\title{
Development of best practices to minimize wound complications after complex tethered spinal cord surgery: a modified Delphi study
}

\author{
Nikita G. Alexiades, MD, ${ }^{1}$ Edward S. Ahn, MD, ${ }^{2}$ Jeffrey P. Blount, MD, ${ }^{3}$ \\ Douglas L. Brockmeyer, MD, ${ }^{4}$ Samuel R. Browd, MD, PhD, ${ }^{5}$ Gerald A. Grant, MD, ${ }^{6}$ \\ Gregory G. Heuer, MD, PhD, ${ }^{7}$ Todd C. Hankinson, MD, MBA, ${ }^{8}$ Bermans J. Iskandar, MD, ${ }^{9}$ \\ Andrew Jea, MD, MHA, ${ }^{10}$ Mark D. Krieger, MD, ${ }^{11}$ Jeffrey R. Leonard, MD, ${ }^{12}$ \\ David D. Limbrick Jr., MD, PhD, ${ }^{13}$ Cormac O. Maher, MD, ${ }^{14}$ Mark R. Proctor, MD, ${ }^{15}$ \\ David I. Sandberg, MD, ${ }^{16}$ John C. Wellons III, MD, MSPH, ${ }^{17}$ Belinda Shao, BA, ${ }^{1,18}$ \\ Neil A. Feldstein, MD, ${ }^{1}$ and Richard C. E. Anderson, MD1
}

1Department of Neurological Surgery, Columbia University Medical Center, New York, New York; ${ }^{2}$ Department of Neurological Surgery, Johns Hopkins University School of Medicine, Baltimore, Maryland; ${ }^{3}$ Department of Neurosurgery, Division of Pediatric Neurosurgery, University of Alabama, Birmingham, Alabama; ${ }^{4}$ Department of Pediatric Neurosurgery, Primary Children's Hospital, University of Utah, Salt Lake City, Utah; ${ }^{5}$ Department of Neurosurgery, University of Washington Seattle Children's Hospital, Seattle, Washington; ${ }^{6}$ Department of Neurosurgery, Stanford University, Stanford, California; ${ }^{7}$ Department of Neurosurgery, Children's Hospital of Philadelphia, Pennsylvania; ${ }^{8}$ Department of Pediatric Neurosurgery, Children's Hospital Colorado, Anschutz Medical Campus, Aurora, Colorado; ' Department of Neurosurgery, University of Wisconsin Hospitals and Clinics, Madison, Wisconsin; ${ }^{10}$ Department of Neurological Surgery, Indiana University School of Medicine, Indianapolis, Indiana; ${ }^{11}$ Department of Neurological Surgery, USC Keck School of Medicine/Children's Hospital of Los Angeles, California; ${ }^{12}$ Department of Neurosurgery, Nationwide Children's Hospital, The Ohio State University College of Medicine, Columbus, Ohio; ${ }^{13}$ Department of Neurological Surgery, Washington University School of Medicine, St. Louis, Missouri; ${ }^{14}$ Department of Neurosurgery, University of Michigan, Ann Arbor, Michigan; ${ }^{15}$ Department of Neurosurgery, Children's Hospital Boston, Harvard Medical School, Boston, Massachusetts; ${ }^{16}$ Department of Neurosurgery, McGovern Medical School/University of Texas Health Science Center, Houston, Texas; ${ }^{17}$ Department of Neurological Surgery, Vanderbilt University Medical Center, Nashville, Tennessee; and ${ }^{18}$ Rutgers New Jersey Medical School, Newark, New Jersey

OBJECTIVE Complications after complex tethered spinal cord (CTSC) surgery include infections and cerebrospinal fluid (CSF) leaks. With little empirical evidence to guide management, there is variability in the interventions undertaken to limit complications. Expert-based best practices may improve the care of patients undergoing cTSC surgery. Here, authors conducted a study to identify consensus-driven best practices.

METHODS The Delphi method was employed to identify consensual best practices. A literature review regarding cTSC surgery together with a survey of current practices was distributed to 17 board-certified pediatric neurosurgeons. Thirty statements were then formulated and distributed to the group. Results of the second survey were discussed during an inperson meeting leading to further consensus, which was defined as $\geq 80 \%$ agreement on a 4-point Likert scale (strongly agree, agree, disagree, strongly disagree).

RESULTS Seventeen consensus-driven best practices were identified, with all participants willing to incorporate them into their practice. There were four preoperative interventions: $(1,2)$ asymptomatic AND symptomatic patients should be referred to urology preoperatively, $(3,4)$ routine preoperative urine cultures are not necessary for asymptomatic AND symptomatic patients. There were nine intraoperative interventions: (5) patients should receive perioperative cefazolin or an equivalent alternative in the event of allergy, (6) chlorhexidine-based skin preparation is the preferred regimen, (7) saline irrigation should be used intermittently throughout the case, (8) antibiotic-containing irrigation should be used fol-

ABBREVIATIONS CSF = cerebrospinal fluid; $\mathrm{CTSC}=$ complex tethered spinal cord; $\mathrm{SSI}=$ surgical site infection; TCS = tethered cord syndrome .

SUBMITTED April 26, 2018. ACCEPTED June 13, 2018.

INCLUDE WHEN CITING Published online September 14, 2018; DOI: 10.3171/2018.6.PEDS18243. 
lowing dural closure, (9) a nonlocking running suture technique should be used for dural closure, (10) dural graft overlay should be used when unable to obtain primary dural closure, (11) an expansile dural graft should be incorporated in cases of lipomyelomeningocele in which primary dural closure does not permit free flow of CSF, (12) paraxial muscles should be closed as a layer separate from the fascia, (13) routine placement of postoperative drains is not necessary. There were three postoperative interventions: (14) postoperative antibiotics are an option and, if given, should be discontinued within 24 hours; (15) patients should remain flat for at least 24 hours postoperatively; (16) routine use of abdominal binders or other compressive devices postoperatively is not necessary. One intervention was prioritized for additional study: (17) further study of additional gram-negative perioperative coverage is needed.

CONCLUSIONS A modified Delphi technique was used to develop consensus-driven best practices for decreasing wound complications after cTSC surgery. Further study is required to determine if implementation of these practices will lead to reduced complications. Discussion through the course of this study resulted in the initiation of a multicenter study of gram-negative surgical site infections in cTSC surgery.

https://thejns.org/doi/abs/10.3171/2018.6.PEDS18243

KEYWORDS tethered spinal cord; surgical site infection; Delphi method; cerebrospinal fluid leak; spine

$\mathrm{T}$ Ethered cord syndrome (TCS) encompasses a disparate group of radiographic findings united around an increasingly well-defined clinical entity. Clinical presentations vary with age and can include cutaneous, neurological, orthopedic, and urological findings depending on a particular pathology and its severity. Causes of tethered spinal cord can be broadly grouped into congenital (primary) tethering or acquired (secondary) causes, such as posttraumatic or postinfectious tethering. ${ }^{1}$ Congenital causes can be further subdivided into a variety of embryologically discrete conditions, including fatty filum, lipomas, dermal sinus tracts, split cord malformations, myelomeningocele, and others. ${ }^{1}$

Specific operative interventions are required for each cause of TCS, with varying levels of complexity ranging from simple filum release to complex lipomyelomeningocele resection and release. Regardless of the underlying pathology, the most common complications following operation for TCS are surgical site infection (SSI) and cerebrospinal fluid (CSF) leakage. 1,9,26,31 Reported complication rates can vary depending on the underlying pathology, for example, from $3.2 \%$ (infection) and $5.9 \%$ (CSF leak) in simple filum release ${ }^{9}$ to $5.6 \%$ and $16.7 \%$ in detethering following myelomeningocele repair ${ }^{20}$ with overall rates between $1 \%$ and $17 \%$ for each complication in all pathologies. ${ }^{26,29,31}$ The literature on this subject is limited to mostly retrospective studies, with a handful of studies on specific strategies to mitigate postoperative CSF leaks. ${ }^{23,24,29}$

Because of the wide variation that exists across centers and surgeons with regard to the techniques used to minimize wound infections and CSF leaks following complex tethered cord release, we first conducted a systematic literature review of existing evidence for a series of pre-, intra-, and postoperative preventative strategies. Given the lack of sufficient empirical evidence available, we used the Delphi method of building consensus among a group of experienced pediatric neurosurgeons to frame best practice statements. Originally developed in the 1960 s by the Rand Corporation, the Delphi method is a technique to build consensus around expert opinions using a structured process of repeated anonymous questionnaires followed by feedback at the culmination of each round. This technique improves on panel or committee approaches by removing direct confrontation while allowing for anonymous expression of expert opinion and criticism. ${ }^{6}$ The modification employed in this and other studies in the medical literature is a culmination of the process in an inperson meeting in which experts can freely discuss topics that approached consensus, allowing for collaboration and modification of statements to gain support. This modified Delphi method has been successfully applied across a number of medical subspecialties, including pediatric scoliosis, in which a similar approach was taken to address the problem of high rates of SSI in a population similar to the pediatric complex tethered cord patients..$^{33,34}$

\section{Methods \\ Focus of Study}

This study was limited to interventions surrounding complex tethered spinal cord surgery in children. We chose to exclude simple filum release because of the significantly reduced complication rate reported in the literature compared to that for other TCS surgery. ${ }^{9,31}$ Furthermore, patients with myelomeningocele were excluded due to the unique aspects of the initial closure of open neural tube defects (e.g., CSF leak and potential infection preoperatively). ${ }^{4,20}$

Our goal was to build consensus around a disparate group of pathologies with an inherently greater risk of wound-related complications (termed "complex tethered spinal cord") for which there is significant clinical variability and uncertainty. The final best practice statements could then be generalized to additional tethered spinal cord release surgeries at each clinician's discretion.

\section{Modified Delphi Study Design}

In order to identify potential strategies to minimize SSI and CSF leaks following complex tethered spinal cord release, a broad literature review was conducted, summarized, and distributed to participants. A list of pre-, intra-, and postoperative interventions was created, and an electronic survey was then generated and distributed to participants to assess general practice patterns (described below). Primary survey responses were collected and analyzed, and statements focusing on these specific perioperative interventions were developed. A second sur- 
vey that included these statements was then distributed to participants to build consensus. Results of the second survey were collected, and statements were modified and revised. A face-to-face meeting was then convened to foster discussion and re-voting on interventions that reached near consensus $(70 \%-79 \%)$. A final document including consensus statements was then generated.

\section{Participants}

Seventeen experienced pediatric neurosurgeons (defined as fellowship trained in pediatric neurosurgery with more than 5 years in clinical practice) from children's hospitals in the United States were identified by the lead author (R.C.E.A.) and recruited as participants for the study. Individuals were selected based on prior collaboration, clinical experience and volume, prior research into pediatric neurosurgical outcomes and complex tethered cord release, and membership in academic pediatric neurosurgical organizations.

\section{Initial Survey}

A 35-item online survey (SurveyMonkey) was distributed to participating pediatric neurosurgeons in July 2017. The survey was segmented into four parts with initial questions covering background information on participants, followed by sections detailing pre-, intra-, and postoperative interventions (Table 1). Participants were initially asked how many years they had been in practice, how many complex tethered cord releases they had done in the last year and in their career, and their estimate of personal rates of postoperative infection and CSF leaks. Participants used a 3-point Likert scale (very willing, somewhat willing, not willing) to indicate how willing they would be to change their current practices based on the results of the current study.

\section{Delphi Round 1}

Using results of the initial survey, we distilled the findings into 30 consensus statements (Table 2). Open-ended questions from the initial survey were adapted and modified into statement form so that a 4-point Likert scale (strongly agree, agree, disagree, strongly disagree) could be used to comment on each statement. While the language and structure of statements were altered, the covered topics were unchanged with some similar statements being merged for brevity given their overlapping scope. The survey was electronically distributed in October 2017, and responses were anonymously collected. Responses with $\geq 80 \%$ agreement (strongly agree and agree) or disagreement (disagree and strongly disagree) were considered to have reached consensus. Responses with near consensus $(70 \%-79 \%)$ were the primary focus of discussion at the face-to-face meeting.

\section{Delphi Round 2}

A face-to-face meeting was held in December 2017 with 14 participants in attendance. All participants used an anonymous audience response system (Poll Everywhere) to enter their responses to statements via their personal smartphone or computer. Responses were tallied
TABLE 1. Electronic survey of current practices

1. Do you routinely refer asymptomatic complex tethered cords to urology preoperatively?

2. Do you routinely refer symptomatic complex tethered cords to urology preoperatively?

3. Do you routinely obtain preoperative urine cultures and treat if positive?

4. Do you use preoperative antibacterial (e.g., chlorhexidine) wash or wipes the night prior to surgery?

5. Do you use prophylactic intravenous cefazolin routinely for surgery?

6. Do you routinely administer prophylactic gram-negative antibiotic coverage during surgery?

7. How long do you continue intravenous antibiotics after surgery?

8. Do you routinely administer steroids perioperatively? If so, for what duration?

9. Do you use chlorhexidine- or Betadine-based scrub and prep?

10. Do you routinely use any irrigation throughout the case, following dural closure, or not at all?

11. Do you use antibiotic irrigation throughout the case, following dural closure, or not at all?

12. Do you place vancomycin powder in the wound prior to closure?

13. Do you use an absorbable or nonabsorbable dural suture?

14. Do you employ a running or interrupted dural closure?

15. If running a dural suture, do you use a locking or nonlocking method?

16. Do you place a dural graft (i.e., Duragen) overlay routinely?

17. Do you routinely sew in an expansile dural graft in cases of lipomyelomeningocele?

18. Do you routinely use a dural sealant (i.e., Duraseal or fibrin glue) following dural closure?

19. Do you close the paraxial muscles as a layer separate from the fascia?

20. Do you use absorbable or nonabsorbable skin sutures?

21. Do you use a running or subcuticular skin closure?

22. Do you routinely leave any drains postoperatively?

23. What type of postoperative dressing do you use?

24. How often do you change dressings postoperatively?

25. Do you place position restrictions on patients postoperatively?

26. How long do you keep patients flat postoperatively?

27. Do you place any position restrictions on patients at discharge?

28. How long do you leave nonabsorbable sutures in place postoperatively? (If used)

29. Do you place patients in an abdominal binder (or other compressive device) postoperatively? If so, for how long?

30. Would you be willing to alter any aspect of your standard practices regarding complex tethered cord releases as a result of evidencebased or consensus-driven findings?

and projected in real time to the participants. Statements that had already reached consensus were briefly reviewed, whereas statements near consensus were discussed in detail and modified to try to achieve consensus. Finalized statements with $\geq 80 \%$ consensus were then incorporated into a consensus statement of proposed best practices. At 


\section{TABLE 2. Consensus round 1 for best practices}

1. Asymptomatic complex tethered cord patients should be referred to urology for preoperative evaluation.

2. Symptomatic complex tethered cord patients should be referred to urology for preoperative evaluation.

3. Routine preoperative urine cultures are not necessary in asymptomatic patients.

4. Routine preoperative urine cultures are not necessary in symptomatic patients.

5. Patients should have chlorhexidine wash performed prior to complex tethered cord release.

6. Patients should receive perioperative cefazolin or an equivalent alternative in the event of allergy.

7. Routine perioperative gram-negative antibiotic coverage is not necessary.

8. Perioperative antibiotics should be continued for 24 hours postoperatively.

9. Steroids should be administered perioperatively.

10. Chlorhexidine-based perioperative skin preparation is the preferred regimen.

11. lodine-based perioperative skin preparation is the preferred regimen.

12. Saline irrigation should be used intermittently throughout the case.

13. Antibiotic-containing irrigation should be used intermittently throughout the case.

14. Antibiotic-containing irrigation should be used following dural closure.

15. Routine use of vancomycin powder in the wound prior to skin closure is not necessary.

16. Nonabsorbable sutures should be used for dural closure.

17. A nonlocking running technique should be used for dural closure.

18. A dural graft overlay should be used when unable to obtain primary dural closure.

19. An expansile dural graft should be incorporated in cases of lipomyelomeningocele in which a primary dural closure does not permit free flow of CSF around the cord.

20. A dural sealant should be used following dural closure.

21. The paraxial muscles should be closed as a layer separate from the fascia.

22. Absorbable sutures should be used for skin closure.

23. Routine placement of postoperative drains is not necessary.

24. Occlusive dressings should be used postoperatively.

25. Postoperative dressings should remain in place until just prior to discharge unless soiled.

26. Patients should remain flat for at least 24 hours postoperatively.

27. Patients should remain flat for at least 48 hours postoperatively.

28. Patients should remain flat for at least 72 hours postoperatively.

29. Position restrictions after discharge are not necessary.

30. Routine use of abdominal binders or other compressive devices postoperatively is not necessary.

the conclusion of the discussion, participants were asked if they would 1) agree to the publication of the consensus statements, 2) implement the consensus statements into their practice, and 3) be willing to participate in multi- center prospective studies to investigate the efficacy of the implementation of the statements.

\section{Results}

\section{Participant Characteristics}

Of the 17 participants invited to participate in the study, $100 \%$ completed the initial survey of current practices. Of these participants, $41.2 \%$ had been in practice for $15-20$ years, $29.4 \%$ for $5-10$ years, $23.5 \%$ for $10-15$ years, and $5.9 \%$ for $>20$ years. Sixty-five percent of participants had performed $>10$ releases in the last year, and $41.2 \%$ of participants had performed $>100$ complex tethered cord releases in their career, with $29.4 \%, 5.9 \%, 5.9 \%$, and $17.7 \%$ having performed $75-100,50-75,25-50$, and $0-25$ releases, respectively. Sixty-five percent of the group estimated CSF leak rates of between $0 \%$ and $5 \%$, whereas $29 \%$ and $6 \%$ of the group estimated rates of $5 \%-10 \%$ and $10 \%-15 \%$, respectively. With respect to estimated wound infection rates, $53 \%$ of the group cited rates between $0 \%$ and $2.5 \%$, whereas $41.2 \%$ reported rates of $2.5 \%-5 \%$ and $5.9 \%$ reported rates of $5 \%-7.5 \%$. All participants estimated rates of CSF leaks and wound infections following complex tethered cord repair that fell within the rates documented in the literature.

\section{Survey of Current Practices}

Of the 29 interventions and preventative practices investigated (Table 1), 6 were employed by $\geq 80 \%$ of participants: $94.1 \%$ of respondents refer symptomatic patients to urology preoperatively, $94.1 \%$ routinely use cefazolin prophylaxis, $94.1 \%$ use irrigation throughout surgery, $100 \%$ use a running dural suture for closure, $82.4 \%$ close the paraxial muscles as a layer separate from the fascia, and $82.4 \%$ use an absorbable suture for skin closure. Moreover, 4 practices were not used by $\geq 80 \%$ of participants: $82.4 \%$ do not place vancomycin powder in the wound prior to closure, $82.4 \%$ do not routinely leave drains in place postoperatively, $94.1 \%$ do not place position restrictions on patients after discharge, and $100 \%$ do not routinely place patients in abdominal binders or other compressive devices postoperatively. All respondents stated that they were willing or somewhat willing to alter aspects of their current practices based on best practice consensus statements.

\section{Delphi Method}

The initial round of 30 proposed consensus statements resulted in $\geq 80 \%$ agreement for the 15 statements listed in Table 3. Ten statements reached near consensus, seven of which were near consensus in agreement (70\%-79\% strongly agreed or agreed) and three of which were near consensus in disagreement $(70 \%-79 \%$ disagreed or strongly disagreed). The statements near consensus in agreement were as follows: routine perioperative gramnegative coverage is not necessary $(76.5 \%)$, routine use of vancomycin powder in the wound prior to closure is not necessary (76.5\%), nonabsorbable sutures should be used for dural closure (76.5\%), absorbable sutures should be used for skin closure (70.6\%), patients should remain flat for at least 48 hours postoperatively (70.6\%), position restrictions after discharge are not necessary (76.5\%), 
TABLE 3. Final consensus best practices to prevent wound complications following complex tethered cord repair

\begin{tabular}{|c|c|c|c|}
\hline \multirow[b]{3}{*}{ Statement } & \multicolumn{3}{|c|}{ Consensus (\%) } \\
\hline & \multicolumn{3}{|c|}{ Strongly } \\
\hline & Total & Agree & Agree \\
\hline $\begin{array}{l}\text { 1. Asymptomatic complex tethered cord } \\
\text { patients should be referred to urology } \\
\text { for preoperative evaluation. }\end{array}$ & 81.25 & 56.25 & 25.00 \\
\hline $\begin{array}{l}\text { 2. Symptomatic complex tethered cord } \\
\text { patients should be referred to urology } \\
\text { for preoperative evaluation. }\end{array}$ & 94.12 & 70.59 & 23.53 \\
\hline $\begin{array}{l}\text { 3. Routine preoperative urine cultures } \\
\text { are not necessary in asymptomatic } \\
\text { patients. }\end{array}$ & 94.12 & 17.65 & 76.47 \\
\hline $\begin{array}{l}\text { 4. Routine preoperative urine cultures are } \\
\text { not necessary in symptomatic patients. }\end{array}$ & 88.23 & 11.76 & 76.47 \\
\hline $\begin{array}{l}\text { 5. Patients should receive perioperative } \\
\text { cefazolin or equivalent alternative in } \\
\text { the event of allergy. }\end{array}$ & 100.0 & 76.47 & 23.53 \\
\hline $\begin{array}{l}\text { 6. Chlorhexidine-based perioperative skin } \\
\text { preparation is the preferred regimen. }\end{array}$ & 82.05 & 23.53 & 58.82 \\
\hline $\begin{array}{l}\text { 7. Saline irrigation should be used inter- } \\
\text { mittently throughout the case. }\end{array}$ & 94.12 & 17.65 & 76.47 \\
\hline $\begin{array}{l}\text { 8. Antibiotic-containing irrigation should } \\
\text { be used following dural closure. }\end{array}$ & 88.24 & 41.18 & 47.06 \\
\hline $\begin{array}{l}\text { 9. A nonlocking running suture technique } \\
\text { should be used for dural closure. }\end{array}$ & 87.5 & 18.75 & 68.75 \\
\hline $\begin{array}{l}\text { 10. A dural graft overlay should be used } \\
\text { when unable to obtain primary dural } \\
\text { closure. }\end{array}$ & 93.75 & 37.50 & 56.25 \\
\hline $\begin{array}{l}\text { 11. An expansile dural graft should be } \\
\text { incorporated in cases of lipomyelo- } \\
\text { meningocele in which a primary dural } \\
\text { closure does not permit free flow of } \\
\text { CSF around the cord. }\end{array}$ & 82.35 & 52.94 & 29.41 \\
\hline $\begin{array}{l}\text { 12. The paraxial muscles should be closed } \\
\text { as a layer separate from the fascia. }\end{array}$ & 88.23 & 11.76 & 76.47 \\
\hline $\begin{array}{l}\text { 13. Routine placement of postoperative } \\
\text { drains is not necessary. }\end{array}$ & 82.35 & 23.53 & 58.82 \\
\hline $\begin{array}{l}\text { 14. Patients should remain flat for at least } \\
24 \text { hours postoperatively. }\end{array}$ & 94.12 & 23.53 & 70.59 \\
\hline $\begin{array}{l}\text { 15. Routine use of abdominal binders or } \\
\text { other compressive devices postopera- } \\
\text { tively is not necessary. }\end{array}$ & 94.12 & 41.18 & 52.94 \\
\hline
\end{tabular}

and occlusive dressings should be used postoperatively (70.6\%). The statements near consensus in disagreement were as follows: steroids should be administered perioperatively (64.7\% disagreed or strongly disagreed), iodinebased perioperative skin preparation is the preferred regimen (64.7\% disagreed or strongly disagreed), and patients should remain flat for at least 72 hours postoperatively (70.6\% disagreed or strongly disagreed).

Five additional statements were indeterminate $(<70 \%$ agreement): patients should have chlorhexidine wash performed prior to complex tethered cord release (35.3\% dis-
TABLE 4. Revisions to selected statements achieving consensus after Delphi round 2

\begin{tabular}{cc}
\hline Initial Statement & $\begin{array}{c}\text { Final Statement } \\
(\% \text { agree or strongly agree })\end{array}$ \\
\hline $\begin{array}{c}\text { Routine perioperative gram- } \\
\text { negative antibiotic coverage } \\
\text { is not necessary. }\end{array}$ & $\begin{array}{c}\text { Further study of additional gram- } \\
\text { negative antibiotic perioperative } \\
\text { coverage is needed. (100\%) }\end{array}$ \\
\hline $\begin{array}{c}\text { Perioperative antibiotics } \\
\text { should be continued for 24 } \\
\text { hours postoperatively. }\end{array}$ & $\begin{array}{c}\text { Postoperative antibiotics are an } \\
\text { option and, if given, should be dis- } \\
\text { continued within 24 hours. (100\%) }\end{array}$ \\
\hline
\end{tabular}

agreed or strongly disagreed), perioperative antibiotics should be continued for 24 hours postoperatively (31.3\% disagreed or strongly disagreed), antibiotic-containing irrigation should be used intermittently throughout the case (31.3\% disagreed or strongly disagreed), a dural sealant should be used following dural closure $(47.1 \%$ disagreed or strongly disagreed), and postoperative dressings should remain in place until just prior to discharge unless soiled (35.3\% disagreed or strongly disagreed).

Statements reaching near consensus were the primary focus of discussion in the subsequent face-to-face meeting. Using the Delphi method, we modified one statement near consensus and one indeterminate statement, and consensus was achieved (Table 4). At the conclusion of the process, 17 statements had consensus, resulting in the current best practice statements (Tables 3 and 4), with the 13 statements that did not have consensus excluded (Table 5). All participants at the face-to-face session agreed to support the publication of the resulting consensus statements.

\section{Discussion}

In this report, we utilized a modified Delphi approach among 17 experienced pediatric neurosurgeons to identify best practices to reduce SSIs and CSF leaks in children undergoing complex tethered spinal cord surgery. We hope that the resulting consensus statements can serve as a platform to decrease variation in clinical practice and lead to further multicenter studies investigating the efficacy of these statements.

As with many other pathologies challenging pediatric neurosurgery, complex tethered spinal cords are infrequent and heterogeneous enough that large-scale, methodologically robust clinical trials are near-impossible to perform. As a result, the quality of the existing evidence guiding current best practices is limited to retrospective series and extrapolation of evidence from other pathologies that may not be appropriately generalized. In areas in which sound evidence is lacking, other research methods have been employed to decrease variance and guide clinical practice. A number of methodologies exist to systematize consensus building, and these have been increasingly applied to the medical field. ${ }^{27,33,34}$ The Delphi method employs the repeated administration of surveys to groups of experts, with discussion and modification of statements after each round to achieve consensus.

Application of the Delphi method provided a framework to greatly increase the number of statements in 
TABLE 5. Interventions to prevent wound complications in complex tethered cord repair that did not reach consensus

\begin{tabular}{|c|c|c|c|c|c|}
\hline \multirow[b]{3}{*}{ Intervention } & \multicolumn{5}{|c|}{ Response (\%) } \\
\hline & \multirow[b]{2}{*}{ Summary } & \multicolumn{3}{|l|}{ Strongly } & \multirow{2}{*}{$\begin{array}{l}\text { Strongly } \\
\text { Disagree }\end{array}$} \\
\hline & & Agree & Agree & Disagree & \\
\hline $\begin{array}{l}\text { 1. Patients should have chlorhexidine wash performed prior to } \\
\text { complex tethered cord release. }\end{array}$ & $35.29 \%$ disagree or strongly disagree & 47.06 & 17.65 & 29.41 & 5.88 \\
\hline 2. Steroids should be administered perioperatively. & $64.7 \%$ disagree or strongly disagree & 5.88 & 29.41 & 35.29 & 29.41 \\
\hline $\begin{array}{l}\text { 3. lodine-based perioperative skin preparation is the preferred } \\
\text { regimen. }\end{array}$ & $64.7 \%$ disagree or strongly disagree & 0.00 & 35.29 & 52.94 & 11.76 \\
\hline $\begin{array}{l}\text { 4. Antibiotic-containing irrigation should be used intermittently } \\
\text { throughout the case. }\end{array}$ & $31.25 \%$ disagree & 25.00 & 43.75 & 31.25 & 0.00 \\
\hline $\begin{array}{l}\text { 5. Routine use of vancomycin powder in the wound prior to skin } \\
\text { closure is not necessary. }\end{array}$ & $23.53 \%$ disagree or strongly disagree & 23.53 & 52.94 & 17.65 & 5.88 \\
\hline 6. Nonabsorbable sutures should be used for dural closure. & $23.53 \%$ disagree & 29.41 & 47.06 & 23.53 & 0.00 \\
\hline 7. A dural sealant should be used following dural closure. & $47.06 \%$ disagree & 11.76 & 41.18 & 47.06 & 0.00 \\
\hline 8. Absorbable sutures should be used for skin closure. & $29.41 \%$ disagree & 11.76 & 58.82 & 29.41 & 0.00 \\
\hline 9. Occlusive dressings should be used postoperatively. & $29.41 \%$ disagree or strongly disagree & 23.53 & 47.06 & 17.65 & 11.76 \\
\hline $\begin{array}{l}\text { 10. Postoperative dressings should remain in place until just prior } \\
\text { to discharge unless soiled. }\end{array}$ & $35.29 \%$ disagree or strongly disagree & 0.00 & 64.71 & 29.41 & 5.88 \\
\hline 11. Patients should remain flat for at least 48 hours postoperatively. & $29.41 \%$ disagree & 29.41 & 41.18 & 29.41 & 0.00 \\
\hline 12. Patients should remain flat for at least 72 hours postoperatively. & $70.59 \%$ disagree or strongly disagree & 11.76 & 17.65 & 52.94 & 17.65 \\
\hline 13. Position restrictions after discharge are not necessary. & $23.53 \%$ disagree & 17.65 & 58.82 & 23.53 & 0.00 \\
\hline
\end{tabular}

which consensus could be reached. The initial survey of current practices identified 6 interventions employed by $\geq$ $80 \%$ of participants. Following the first round of consensus building, a total of 15 statements had consensus, and after the face-to-face meeting an additional 2 statements had consensus. One intervention that reached the threshold of $\geq 80 \%$ on the initial survey-absorbable sutures should be used for skin closure-ultimately did not reach consensus following further discussion and repeat survey.

\section{Preoperative Best Practice Statements}

Four preoperative interventions reached consensus, one following the initial survey and three following the first consensus-building round. The group reached consensus on preoperative urological evaluation for symptomatic patients after the initial survey $(94.22 \%$ agree or strongly agree) and for asymptomatic patients following the first consensus survey (81.25\% agree or strongly agree). One benefit of formal urological evaluation lies in establishing a perioperative plan for the management of incontinence to prevent potential soiling of the operative site. While consensus preexisted regarding overtly symptomatic patients, consensus on clinically asymptomatic patients was likely influenced by further review of the current literature suggesting that significant proportions of clinically asymptomatic patients have abnormal urodynamic findings that may be unmasked further in the perioperative period. ${ }^{17,21}$ The group also reached consensus on the lack of current indications for routine preoperative urine cultures in both clinically symptomatic (88.23\%) and asymptomatic $(94.12 \%)$ patients. While preoperative urinary tract infections are an identified modifiable risk factor in the neuromuscular pediatric spinal deformity surgery population, ${ }^{15,32}$ expert opinion among our group held that this was unnecessary in the complex tethered spinal cord population.

\section{Intraoperative Best Practice Statements}

A total of nine intraoperative interventions were identified as consensus best practices following the first Delphi survey round. The interventions identified are a mix of practical modifications without proven risk to patients along with interventions drawn from the general surgical and neurosurgical literature with a strong evidence base. All participants agreed (100\%) that cefazolin or an equivalent alternative in the event of allergy should be administered perioperatively. While not novel as a best practice recommendation, consensus here validates the group's compliance with national evidence-based recommendations. ${ }^{5}$ The group reached consensus $(82.05 \%)$ on the use of chlorhexidine-based skin preparation as the preferred regimen, citing evidence from other populations and the absent risk of ocular injury given the lumbosacral operative site. ${ }^{3,11,13}$ Consensus regarding the use of saline irrigation throughout the operation was preexisting (94.12\%), and following the first Delphi round, consensus was reached regarding the use of antibiotic-containing irrigation following dural closure (88.24\%). No specific antibiotic in the irrigation could be recommended by the group as evidence supporting this practice is derived from the general neurosurgical and spinal surgery literature..$^{16,19}$ Consensus could not be reached on the use of antibioticcontaining irrigation throughout the case including prior to dural closure, with participants citing concerns regarding the application of high antibiotic doses directly to the neural elements without adequate safety data.

Three dural closure techniques were identified and had consensus: the use of a nonlocking running stitch $(87.5 \%$ 
agree or strongly agree), the use of a dural graft overlay when unable to obtain primary dural closure $(93.75 \%$ agree or strongly agree), and the incorporation of an expansile dural graft in cases of lipomyelomeningocele in which primary dural closure does not permit free flow of CSF around the cord (82.35\% agree or strongly agree). The recommendation of suture technique stems largely from the group's anecdotal evidence as there are few studies on the strength of closure provided by each technique, none of which was performed in humans. ${ }^{8,10}$ The use of dural graft overlays is supported by a number of studies in the neurosurgical literature, though none has directly examined their use following tethered cord release..$^{22,30}$ The incorporation of expansile dural grafts in lipomyelomeningocele repair has been associated with good neurological outcomes and low rates of retethering, but their role in decreasing wound complications is less clear. Thus, its recommendation as a best practice relates more to the overall care of these patients than the strength of existing evidence for this practice. ${ }^{25,26}$ Closure of the paraxial muscles as a layer separate from the fascia had consensus on the initial survey likely based on several early studies in the complex tethered cord literature supporting multilayer soft-tissue closure as a means of decreasing CSF leak rates. ${ }^{2,36}$ Following the initial Delphi round, consensus was also reached on the recommendation against the routine placement of postoperative drains $(82.35 \%$ agree or strongly agree) with the group citing a lack of evidence for their benefit.

\section{Postoperative Best Practice Statements}

Two postoperative interventions achieved early consensus following the initial Delphi round. Ample studies have examined the optimal duration that patients should remain flat in the context of simple tethered cord, but no quality prospective studies have analyzed this factor for complex tethered cords. ${ }^{23,24,28,29}$ Consensus was reached for a minimum duration of 24 hours of flat positioning postoperatively (94.14\% agree or strongly agree). A significant proportion of the group kept patients flat for 48 hours or more, but not at a level sufficient for consensus. Given the potential implications for both wound complications and length of stay, this topic may present an opportunity for a future multicenter study. Although some practitioners place patients in compressive abdominal binders to counteract potential CSF egress, limited data exist on the practice aside from case reports. ${ }^{18}$ As a result, the group reached consensus recommending against the routine use of abdominal binders or other compressive devices postoperatively (94.12\% agree or strongly agree).

Two additional consensus statements were agreed on following the final Delphi round. The unknown prevalence of postoperative gram-negative infections following complex tethered spinal cord surgery coupled with evidence from the neuromuscular scoliosis population prompted modification of the statement that "routine perioperative gram-negative antibiotic coverage is not necessary" to the broad statement that "further study of additional gramnegative antibiotic perioperative coverage is needed." Rather than making a strong recommendation against the practice given the paucity of evidence, the group elected to recommend further study via a multicenter collaboration to inform future recommendations. In addition, the panel believed that a strong recommendation regarding the use and duration of postoperative antibiotics could lead to antibiotic overuse and a challenge to established recommendations. Consensus was reached for the modified statement, "postoperative antibiotics are an option and, if given, should be discontinued within 24 hours." Several statements with evidence from other surgical populations ultimately eluded consensus including those involving the use of preoperative chlorhexidine wash and dressing type and duration. ${ }^{7,12-14,35}$

\section{Study Limitations and Future Studies}

The primary limitations of this study are that these best practice statements are the product, not of empirical evidence, but of a consensus of expert opinion. While the group of experts selected to participate included a high level of experience, all of the experts represent academic pediatric neurosurgeons based in the United States. This relative homogeneity in the population of experts may limit the generalizability of the statements to the broader pediatric neurosurgical population worldwide given the geographic bias in practice patterns. Furthermore, only pediatric neurosurgeons were included in the expert group given the primary focus on topics related to surgical techniques, but this led to a lack of input by other key stakeholders in SSI prevention, such as pediatric infectious disease specialists. Additionally, while the Delphi model assumes that the consensus of groups will lead to better decisions, ignorance and misinformation have the potential to propagate through faulty group dynamics. There is also no preexisting evidence that widespread implementation of these best practices will lead to decreased rates of SSIs and CSF leaks following tethered spinal cord repair as this approach requires ex post facto validation.

The strengths of this approach are inherently linked to its limitations. By design, the focus of this study was an area in which quality empirical evidence is lacking and individual practices are rarely evidence based. By leveraging the experience of a panel of experts, common practices are more likely to be valid than those used by individuals. Perhaps the most important strength identified is the potential of this approach to identify important areas of future collaborative research built within the multicentric infrastructure of the initial Delphi group. Questions generated through the course of this study prompted the acquisition of pilot data identifying higher rates of gram-negative bacteria in complex tethered cord release SSIs than previously reported. As a result, a multicenter study of the incidence of gram-negative SSIs in the complex tethered cord population was planned and initiated. The Delphi approach serves both to identify questions ripe for multicenter collaboration and to establish a ready framework of centers equipped to participate in studies to answer such questions.

\section{Conclusions}

The statements presented here represent an important application of consensus-building techniques to identify 
best practices in the pediatric neurosurgical literature. While not all encompassing, the 17 final consensus statements can be incorporated into the care of complex tethered cord patients to reduce practice variability and to potentially decrease rates of wound complications. The participants have agreed to implement the best practices and to participate in targeted studies of several interventions that did not reach consensus. By minimizing variability not based in evidence by using such best practices, we may incrementally improve the care of patients afflicted with complex tethered cords and further identify areas that are both high impact and accessible to methodologically robust clinical studies.

\section{References}

1. Agarwalla PK, Dunn IF, Scott RM, Smith ER: Tethered cord syndrome. Neurosurg Clin N Am 18:531-547, 2007

2. Arad E, Barnea Y, Gur E, Amir A, Leshem D, Zaretski A, et al: Paravertebral turnover flaps for closure of large spinal defects following tethered cord repair. Ann Plast Surg 57:642-645, 2006

3. Bever GJ, Brodie FL, Hwang DG: Corneal injury from presurgical chlorhexidine skin preparation. World Neurosurg 96:610.e1-610.e4, 2016

4. Bowman RM, Mohan A, Ito J, Seibly JM, McLone DG: Tethered cord release: a long-term study in 114 patients. J Neurosurg Pediatr 3:181-187, 2009

5. Bratzler DW, Dellinger EP, Olsen KM, Perl TM, Auwaerter PG, Bolon MK, et al: Clinical practice guidelines for antimicrobial prophylaxis in surgery. Am J Health Syst Pharm 70:195-283, 2013

6. Brown BB: Delphi Process: A Methodology Used for the Elicitation of Opinions of Experts. Santa Monica, CA: Rand Corporation, 1968 (https://www.rand.org/pubs/papers/ P3925.html) [Accessed July 20, 2018]

7. Bryce E, Wong T, Forrester L, Masri B, Jeske D, Barr K, et al: Nasal photodisinfection and chlorhexidine wipes decrease surgical site infections: a historical control study and propensity analysis. J Hosp Infect 88:89-95, 2014

8. Cain JE Jr, Dryer RF, Barton BR: Evaluation of dural closure techniques. Suture methods, fibrin adhesive sealant, and cyanoacrylate polymer. Spine (Phila Pa 1976) 13:720-725, 1988

9. Chern JJ, Tubbs RS, Patel AJ, Gordon AS, Bandt SK, Smyth $\mathrm{MD}$, et al: Preventing cerebrospinal fluid leak following transection of a tight filum terminale. J Neurosurg Pediatr 8:35-38, 2011

10. Dafford EE, Anderson PA: Comparison of dural repair techniques. Spine J 15:1099-1105, 2015

11. Darouiche RO, Wall MJ Jr, Itani KM, Otterson MF, Webb AL, Carrick MM, et al: Chlorhexidine-alcohol versus povidone-iodine for surgical-site antisepsis. N Engl J Med 362:18-26, 2010

12. Dumville JC, Gray TA, Walter CJ, Sharp CA, Page T, Macefield R, et al: Dressings for the prevention of surgical site infection. Cochrane Database Syst Rev 12:CD003091, 2016

13. Dumville JC, McFarlane E, Edwards P, Lipp A, Holmes A, Liu Z: Preoperative skin antiseptics for preventing surgical wound infections after clean surgery. Cochrane Database Syst Rev (4):CD003949, 2015

14. Glennie RA, Dea N, Street JT: Dressings and drains in posterior spine surgery and their effect on wound complications. J Clin Neurosci 22:1081-1087, 2015

15. Hatlen T, Song K, Shurtleff D, Duguay S: Contributory factors to postoperative spinal fusion complications for children with myelomeningocele. Spine (Phila Pa 1976) 35:12941299,2010
16. Hayashi T, Shirane R, Yokosawa M, Kimiwada T, Tominaga T: Efficacy of intraoperative irrigation with saline for preventing shunt infection. J Neurosurg Pediatr 6:273-276, 2010

17. Hsieh MH, Perry V, Gupta N, Pearson C, Nguyen HT: The effects of detethering on the urodynamics profile in children with a tethered cord. J Neurosurg Pediatr 105:391-395, 2006

18. Leis AA, Leis JM, Leis JR: Pseudomeningoceles: a role for mechanical compression in the treatment of dural tears. Neurology 56:1116-1117, 2001

19. Malis LI: Prevention of neurosurgical infection by intraoperative antibiotics. Neurosurgery 5:339-343, 1979

20. Mehta VA, Bettegowda C, Ahmadi SA, Berenberg P, Thomale UW, Haberl EJ, et al: Spinal cord tethering following myelomeningocele repair. J Neurosurg Pediatr 6:498-505, 2010

21. Meyrat BJ, Tercier S, Lutz N, Rilliet B, Forcada-Guex M, Vernet $\mathrm{O}$ : Introduction of a urodynamic score to detect preand postoperative neurological deficits in children with a primary tethered cord. Childs Nerv Syst 19:716-721, 2003

22. Narotam PK, José S, Nathoo N, Taylon C, Vora Y: Collagen matrix (DuraGen) in dural repair: analysis of a new modified technique. Spine (Phila Pa 1976) 29:2861-2869, 2004

23. Ogiwara H, Joko M, Takado M, Uematsu K, Kameda M, Sasaki N, et al: Duration of the horizontal decubitus position for prevention of cerebrospinal fluid leakage following transection of a tight filum terminale. J Neurosurg Pediatr 15:461-464, 2015

24. Ogiwara H, Morota N, Joko M: Duration of horizontal decubitus after section of a tight filum terminale as a means to prevent cerebrospinal fluid leakage. Surg Neurol Int 3:113, 2012

25. Pang D, Zovickian J, Oviedo A: Long-term outcome of total and near-total resection of spinal cord lipomas and radical reconstruction of the neural placode: part I-surgical technique. Neurosurgery 65:511-529, 2009

26. Pang D, Zovickian J, Oviedo A: Long-term outcome of total and near-total resection of spinal cord lipomas and radical reconstruction of the neural placode, part II: outcome analysis and preoperative profiling. Neurosurgery 66:253-273, 2010

27. Pezold ML, Pusic AL, Cohen WA, Hollenberg JP, Butt Z, Flum DR, et al: Defining a research agenda for patientreported outcomes in surgery: using a Delphi survey of stakeholders. JAMA Surg 151:930-936, 2016

28. Poonia S, Graber S, Corbett Wilkinson C, O'neill BR, Handler MH, Hankinson TC: Outcome of hospital discharge on postoperative Day 1 following uncomplicated tethered spinal cord release. J Neurosurg Pediatr 17:651-656, 2016

29. Shahjouei S, Hanaei S, Habibi Z, Hoseini M, Ansari S, Nejat F: Randomized clinical trial of acetazolamide administration and/or prone positioning in mitigating wound complications following untethering surgeries. J Neurosurg Pediatr 17:659-666, 2016

30. Stendel R, Danne M, Fiss I, Klein I, Schilling A, Hammersen $S$, et al: Efficacy and safety of a collagen matrix for cranial and spinal dural reconstruction using different fixation techniques. J Neurosurg 109:215-221, 2008

31. Thuy M, Chaseling R, Fowler A: Spinal cord detethering procedures in children: a 5 year retrospective cohort study of the early post-operative course. J Clin Neurosci 22:838-842, 2015

32. Vitale MG, Mackenzie SWG II, Matsumoto H, Lee C, Cody SR, Corona J, et al: Surgical site infection following spinal instrumentation for scoliosis: lessons learned from an multicenter analysis of 1352 spinal instrumentation procedures for scoliosis: paper \#32. Spine (Phila Pa 1976) 2011 SRS Suppl:71, 2011 (Abstract)

33. Vitale MG, Riedel MD, Glotzbecker MP, Matsumoto H, Roye 
DP, Akbarnia BA, et al: Building consensus: development of a Best Practice Guideline (BPG) for surgical site infection (SSI) prevention in high-risk pediatric spine surgery. J Pediatr Orthop 33:471-478, 2013

34. Vitale MG, Skaggs DL, Pace GI, Wright ML, Matsumoto H, Anderson RC, et al: Best practices in intraoperative neuromonitoring in spine deformity surgery: development of an intraoperative checklist to optimize response. Spine Deform 2:333-339, 2014

35. Webster J, Osborne S: Preoperative bathing or showering with skin antiseptics to prevent surgical site infection. Cochrane Database Syst Rev (2):CD004985, 2015

36. Zide BM, Epstein FJ, Wisoff J: Optimal wound closure after tethered cord correction. Technical note. J Neurosurg 74:673-676, 1991

\section{Disclosures}

Dr. Limbrick receives support from Medtronic Inc. and Microbot Medical Inc. for non-study-related clinical or research efforts.

\section{Author Contributions}

Conception and design: Alexiades, Anderson. Acquisition of data: Alexiades. Analysis and interpretation of data: Alexiades. Drafting the article: Alexiades. Critically revising the article: Feldstein, Anderson. Reviewed submitted version of manuscript: Ahn, Blount, Brockmeyer, Browd, Grant, Heuer, Hankinson, Iskandar, Jea, Krieger, Leonard, Limbrick, Maher, Proctor, Sandberg, Wellons, Shao, Feldstein, Anderson. Study supervision: Feldstein, Anderson.

\section{Supplemental Information}

\section{Previous Presentations}

Portions of this work were presented in poster form at the AANS/ CNS Section on Pediatric Neurological Surgery Annual Meeting held in Houston, Texas, on November 28-December 1, 2017.

\section{Correspondence}

Nikita G. Alexiades: The Neurological Institute of New York, New York, NY.nga2109@cumc.columbia.edu. 